\title{
Sector Design and Boundary Change Considerations for Flexible Airspace Management
}

\author{
Paul U. Lee ${ }^{1}$, Thomas Prevot ${ }^{2}$, Jeffrey Homola ${ }^{3}$, Hwasoo Lee ${ }^{3}$, and Angela Kessell ${ }^{1}$ \\ SJSU / NASA Ames Research Center, Moffett Field, CA, 94035 \\ Nancy Smith ${ }^{4}$ \\ NASA Ames Research Center, Moffett Field, CA, 94035
}

\begin{abstract}
In Next Generation Air Transportation System (NextGen) operations, we expect that the demand-capacity balance can be achieved by selectively managing the airspace capacity in conjunction with managing the traffic demand. In Flexible Airspace Management (FAM), the airspace complexity can be assessed a few hours ahead in order to identify sectors that could exceed their defined traffic threshold as well as sectors that are under-utilized. Using various airspace optimization algorithms, airspace can be reconfigured to manage the existing traffic demand without moving aircraft away from their original user-preferred routes. A human-in-the-loop simulation study was conducted in 2009 to assess the impact of airspace reconfiguration on the controllers. The results from the objective data found that the acceptability of the boundary change and the associated workload were mainly affected by airspace volume change and aircraft that changed ownership. However, observations and subjective feedback have suggested that other cognitively-driven factors, such as spatial relationships between upstream/downstream sectors, may also play a role, especially in traffic situations where the airspace has only a few aircraft that change ownership but still has a high degree of airspace complexity associated with the reconfiguration. In this paper, we identify these factors and discuss the human factors issues that should be considered in designing the airspace and airspace transitions.
\end{abstract}

\begin{tabular}{|c|c|}
\hline & Nomenclature \\
\hline $4-D$ & $=$ four dimensional ( 3 spatial dimensions plus time $)$ \\
\hline$A N S P$ & $=$ Air Navigation Service Provider \\
\hline$B C$ & $=$ boundary change \\
\hline$D A C$ & $=$ Dynamic Airspace Configuration \\
\hline Data Comm & $=$ data communication \\
\hline$F A A$ & $=$ Federal Aviation Administration \\
\hline FAM & $=$ Flexible Airspace Management \\
\hline$H A A$ & $=$ High Altitude Airspace \\
\hline HITL & $=$ human-in-the-loop \\
\hline NASA & $=$ National Aeronautics and Space Administration \\
\hline NEXTGEN & $=$ Next Generation Air Transportation System \\
\hline$R A$ & $=$ radar associate \\
\hline$S M E$ & $=$ subject matter expert \\
\hline$T B O$ & $=$ Trajectory-Based Operations \\
\hline$T M U$ & $=$ Traffic Management Unit \\
\hline TRACON & $=$ Terminal Radar Approach Control \\
\hline
\end{tabular}

\footnotetext{
${ }^{1}$ Senior Research Associate, Human Systems Integration, MS 262-4.

2 Senior Research Engineer, Human Systems Integration, MS 262-4.

${ }^{3}$ Research Associate, Human Systems Integration, MS 262-4.

${ }^{4}$ Senior Research Psychologist, Human Systems Integration, MS 262-4. 1
}

American Institute of Aeronautics and Astronautics 


\section{Background}

A fundamental aspect of air traffic management is to balance traffic demand with existing airspace capacity. On any given day, various Air Navigation Service Providers (ANSPs) in the air traffic organizations (e.g. Command Center, Traffic Management Units (TMUs), En route, TRACON, and Tower facilities) work together to determine whether the current and forecasted airspace capacity can accommodate the traffic demand. If demand is expected to exceed capacity (e.g., due to weather-related congestion, controller workload, etc.), then traffic flows are restricted using diverse methods such as miles-in-trail, ground delay program, and playbook routes, all of which result in delays and costs to the users.

In modernizing the future air transportation system, increased capacity/throughput, better flight management, and improved flight and system efficiency are among the main drivers for the Next Generation Air Transportation System (NextGen) ${ }^{1}$. In mid-term NextGen operations, we expect that the demand-capacity balance can be achieved by selectively managing the airspace capacity in conjunction with managing the traffic demand. In Flexible Airspace Management (FAM), the airspace size, shape, and overall configuration can be adjusted dynamically to meet the changing traffic demand or airspace congestions. The traffic demand can be calculated up to two hours into the future to identify sectors that could exceed the traffic threshold as well as sectors that are under-utilized. FAM is a component of a research focus area at the National Aeronautics and Space Administration (NASA) called Dynamic Airspace Configuration (DAC) that examines the restructuring of airspace, flexible airspace reconfiguration, and generic airspace design ${ }^{2}$. Using various airspace optimization algorithms, airspace can be reconfigured to manage the existing traffic demand without moving aircraft away from existing routes, resulting in fewer delays. A number of airspace optimization algorithms are currently being explored to find the optimal reconfiguration options for the airspace ${ }^{3-6}$.

The FAM concept is also a part of the Federal Aviation Administration's (FAA) NextGen implementation plan. The concept was initially proposed as a component of the High Altitude Airspace (HAA) concept ${ }^{7}$, but has since been expanded to all airspace classes. In this paper, we describe the airspace reconfiguration studies that have so far targeted the operational environment in the HAA concept, in which the airspace is expected to have aircraft fully equipped with air-ground data communication (Data Comm) conducting Trajectory-Based Operations (TBO) in a relatively simple, low complexity traffic environment (e.g., mostly level flight with little local knowledge needed). Mid-term HAA concept is expected to be more responsive to changes in demand due to improved automation and communication. High altitude sectors are expected to have the ability to modify airspace boundaries in predetermined configurations to accommodate predicted traffic flows. Adjustments to airspace are expected to make use of under-utilized airspace to maintain throughput and to have less impact on users than the traditional traffic flow management tools of ground stops, delays, and reroutes.

\section{Exploring the Feasibility of Flexible Airspace Reconfiguration}

One of the main human-system issues or tasks related to dynamic airspace reconfiguration is identifying the necessary operational procedures and guidelines that would be required to handle the transition from one configuration to another. A better understanding of the human ability to handle the transition is needed. Some of the fundamental questions related to airspace changes and their impact on the controllers are:

- Which airspace-related factors (e.g., airspace volume change, number of aircraft affected by the boundary change, etc.) significantly impact controllers during a boundary change?

- How often can airspace be changed?

- When (e.g., number of aircraft in the sector) is airspace change feasible?

A human-in-the-loop (HITL) simulation was conducted in the Airspace Operations Laboratory at the NASA Ames Research Center in 2009 to address some of the questions posed above ${ }^{8,9}$. Traffic scenarios with varying types and severity of boundary changes (BCs) were used to test their impact on the controllers. The "severity" of the BCs was determined by both subjective assessment and objective metrics such as airspace volume change, number of aircraft that changed sector ownership, etc. For each boundary change, airspace-related metrics such as number of aircraft and various task loads (e.g., number of handoffs, pointouts, etc.) were compared against subjective metrics such as workload and acceptability, as well as the safety implications in terms of losses of separation and other operational deviations.

American Institute of Aeronautics and Astronautics 


\section{A. Experiment Design}

Four en-route sectors from Kansas City Center (ZKC) were chosen as the test airspace and basis for the boundary configurations (see Figure 1). Two traffic scenarios were developed for these sectors with traffic load imbalances between the sectors constructed in order to create an environment where boundary changes or demand reduction would be necessary. These scenarios were then used to create aircraft position data, which in turn served as input for three algorithms that were chosen for this $\operatorname{study}^{3-6}$ to generate various airspace configurations to minimize traffic overload in the congested sectors.

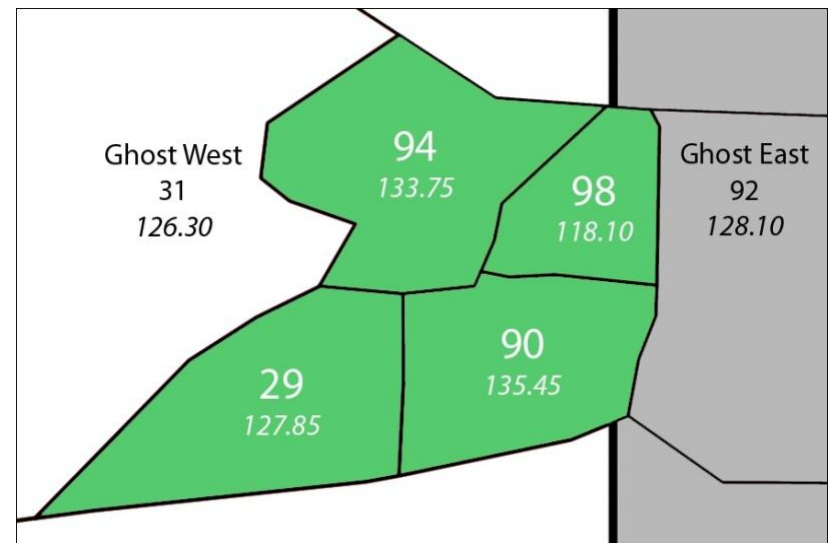

Figure 1. Four test sectors in ZKC

Figure 2 illustrates an example of boundary configurations that were created by the three algorithms. Three airspace reconfiguration algorithms were selected based upon their approach and aggressiveness related to the magnitude of the sector boundary change relative to the initial sector boundaries and the changes were labeled as Low, Medium, and High accordingly ${ }^{4-6}$. A Baseline condition with no BCs was included in the study in order to create a comparison condition that establishes the baseline workload and other performance metrics. In total, the experiment consisted of four test conditions.

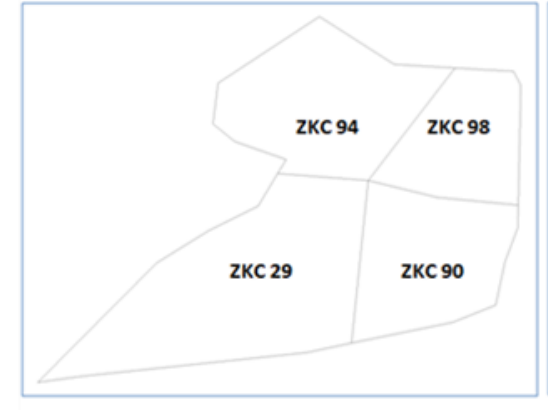

Low Severity

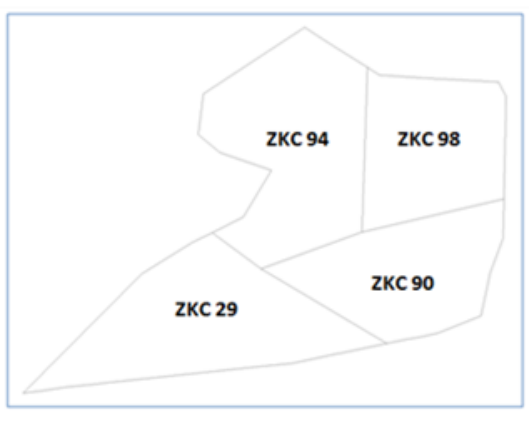

Medium Severity

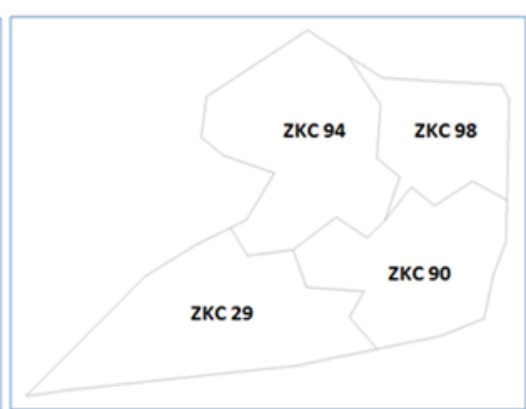

High Severity

Figure 2. Example of boundary changes in the three boundary change conditions

For this study's operational environment, a number of technological assumptions were made that were in line with what is envisioned as part of HAA. This included 4-D trajectory based operations, Data Comm, automatic transfer-of-communication, conflict probe, trial planning, and automated conflict detection/resolution capabilities. Detailed descriptions of these technologies are reported in Ref. 8.

The study was conducted over the course of two weeks and included four test participants and a team of confederate controllers. The test participants served as single radar controllers for the selected sectors, and the confederate controllers filled the roles of an area supervisor, radar associates (RAs), and "ghost" controllers responsible for all of the aircraft outside of the test airspace. A total of sixteen within-subjects data collection runs were conducted across the four test conditions: Baseline (no boundary changes), Low, Medium, and High severity boundary changes. For non-Baseline runs, there was a high frequency of boundary changes (i.e. three BCs in an 
hour). The interval between the BCs was varied between five to thirty minutes and the timing of the changes occurred at times when the traffic volume was high. Both the high frequency of the changes and the adverse traffic situations were used in an effort to test the limits of sector boundary changes in terms of change frequency, volume, and the number of aircraft that change sectors due to the boundary changes. Three minutes prior to the boundary change, participants received a preview of the change, at which point they began making the necessary preparations for the new sector boundary. The area supervisor made decisions on the allocation of RAs to assist overloaded sectors. After the BC, RAs continued to help the radar controllers to become acclimated to the new sector boundaries, normally lasting for a few minutes afterward. For the purposes of the analysis, we defined the "BC duration" to be the time in which the controllers were able to preview the new sector boundaries (i.e., 3 minutes prior a $\mathrm{BC}$ ) to 3 minutes after the $\mathrm{BC}$.

\section{B. Results}

Our initial hypothesis on the impact of sector boundary changes on the controllers was that the BCs would cause high workload whenever the airspace configuration change resulted in an increase in controller tasks. For example, if a large number of aircraft changed ownership from one sector to another during the change, controllers would need to initiate handoffs to change the aircraft ownership or to initiate pointouts when aircraft passed near or through a small portion of an adjacent sector, resulting in a greater number of controller tasks and higher workload.

We also identified other factors we expected to be correlated with increased workload at the BCs. These factors included large changes in sector volume (either due to changes in the sector size or geometry), poor sector design (e.g., sectors that require lots of pointouts, thin sectors with short transit time, etc.), and sectors that cut across traffic flows. Detailed results from this study are available in Ref. 8 and 9 but a high-level summary of the results are provided in the following section.

\section{Workload}

For each boundary change, metrics such as airspace volume change, number of aircraft, and various task loads (e.g., handoffs, pointouts, etc.) were correlated with workload ratings. Hierarchical stepwise regression narrowed the explanatory variables for overall workload during BCs down to airspace volume change, aircraft count, and number of late handoff acceptances ${ }^{9,10}$. Normally aircraft count is one of the main predictors of workload, so it is notable that airspace volume change was a better predictor for the controller workload during airspace reconfiguration than aircraft count. Controller task loads, such as number of handoffs and pointouts, also correlated with the explanatory variables described above as well as the severity of the BCs. Participants' subjective feedback also mirrored the objective metrics. When the participants were asked to identify the factors that created high workload situations during the BCs, they cited heavy overall traffic volume, large number of aircraft ownership transfers, increased number of required pointouts, and high concentrations of aircraft in one area that resulted in overlapping datablocks. Interestingly, high frequency of boundary changes (between 5 to 30 minutes) was not a factor that contributed to high workload in either the objective data or subjective feedback.

\section{Acceptability and Operational Errors/Deviations}

Similar to the workload results, numerous metrics were correlated with acceptability ratings. Hierarchical stepwise regression was used to narrow the explanatory variables for the acceptability of BCs. Hierarchical stepwise regression of the acceptability ratings identified a single factor, aircraft gained/lost, as the strongest predictor of acceptability.

Participant feedback on the acceptability of BCs identified factors and situations in which certain boundary changes were unacceptable even when the workload was relatively moderate. The factors in the $\mathrm{BC}$ acceptability ratings captured some of the factors that we originally hypothesized would impact the workload. These factors were large changes in sector size/geometry, bad sector design, excessive coordination due to a large number of pointouts and handoffs during BCs, too much/not enough airspace, and sector shape that did not align with the traffic flow. Objective data on the amount of volume change during BCs, mean sector transit time, and the number of pointouts/handoffs was consistent with the subjective feedback and corroborated the results. BCs with large shifts in sector volume resulted in a correspondingly large number of aircraft that changed ownership, resulting in high workload and low acceptability. Depending on the geometry of the BCs, some sectors needed to initiate and accept handoffs with multiple adjacent sectors, increasing the cognitive difficulty in transitioning the aircraft. Figure 3 illustrates this point. In Figure 3, the BC changes Sectors 1 through 4 significantly but the changes result in the greatest difficulty for Sector 4 , which inherits space from all three adjoining sectors.

American Institute of Aeronautics and Astronautics 


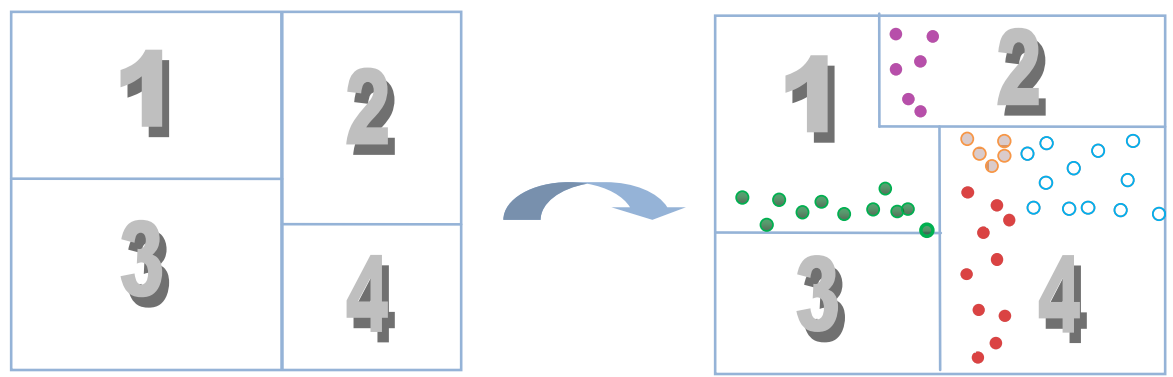

Figure 3. Aircraft in transition due to a boundary change

In this figure, aircraft that change ownership during the $\mathrm{BC}$ are represented by dots. Their colors represent a change in ownership between different sector pairs. The figure shows that Sector 4 has three different colors of aircraft in transition, indicating that this controller needs to coordinate with all three adjacent sectors to successfully change aircraft ownership in the sector, resulting in a more difficult transition compared to the other three sectors.

More severe BCs also resulted in more operational deviations. BCs with large volume changes made it difficult for controllers to transition ownership of certain aircraft during the $\mathrm{BC}$ in a timely manner. Incorrect handling of these aircraft resulted in initiating handoffs too late (i.e., after the aircraft had already exited the sector) or late acceptance of the handoffs (i.e., the handoff was initiated correctly but was accepted after the aircraft had entered the sector). The operational deviations seemed to be often due to cognitive complexities and indecisions created by aircraft that traveled a large distance between the current and future sector boundaries. Figure 4 illustrates this point.

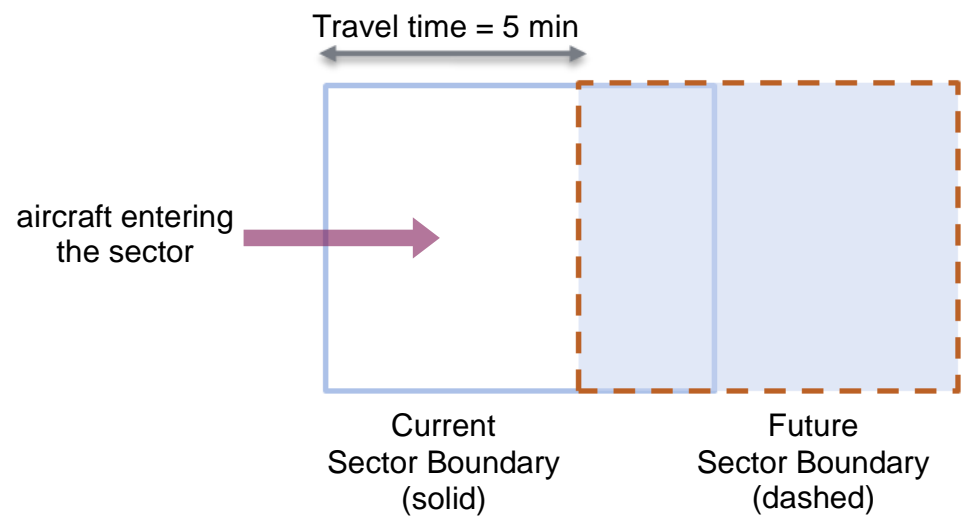

Figure 4. Operational deviation due to large volume change during airspace reconfiguration

In Figure 4, an aircraft enters a sector when the airspace reconfiguration is expected to happen within the next minute. Once the boundary changes, the aircraft is no longer in the new sector (box with dashed lines) and will need to fly at least 4 more minutes before entering the sector again. In such situations, the controllers either handed the aircraft back to the upstream sector and then received the handoff again for the second time in about 4 minutes (thereby creating unnecessary workload) or let the aircraft fly through the upstream sector while keeping the Data Comm / voice frequency control of the aircraft (resulting in a potential safety issue). However, the situation sometimes led the controllers to let the aircraft to approach the sector boundary without accepting handoffs when it seemed that the reconfiguration would occur before the aircraft entered the current sector, resulting in operational deviations when the judgment was incorrect and the aircraft penetrated the sector before the boundary change. These types of operational deviations occurred more frequently as the BC severity increased. In contrast, separation violations occurred across all conditions (e.g. between three to five times per condition) due to the unfamiliarity of the FAM operations but unlike operational deviations, they did not occur more frequently as the BC severity increased.

An interesting and positive outcome of the study was that the participants successfully managed many of the challenging airspace boundary changes that they considered unacceptable due to factors such as poor sector design and/or large changes in sector geometry. This finding suggests some flexibility in allowing more radical changes in sector design during airspace reconfiguration that might be desirable in terms of maximizing the benefits of the

5

American Institute of Aeronautics and Astronautics 
reconfiguration. One significant caveat in the finding, however, was that during many of the airspace boundary changes, participants could not maintain situation awareness of the traffic without the advanced conflict detection tool. Automated transfer-of-communication was also an essential prerequisite that relieved controllers from memorizing and/or searching for frequency information during the boundary changes.

\section{Other Sector Design and Boundary Change Considerations}

In contrast to the prior papers on our 2009 study $^{8-10}$, this paper focuses on the implications of the results in terms of feasible sector design and boundary change considerations for the FAM. Over-the-shoulder observations of the operations and feedback from the subject matter experts (SMEs) on the 'goodness' of the airspace design have suggested that other cognitively-driven factors, such as the spatial relationship between upstream/downstream sectors, may also play a role when there is a high degree of airspace complexity associated with the reconfiguration. In this paper, we identify these factors and discuss the human factors issues that should be considered in designing the airspace and airspace transitions.

We gathered qualitative feedback on the airspace designs and reconfigurations from SMEs prior to the study as well as from the participants during the study. Prior to the study, the SMEs who gave us feedback were retired controllers who were familiar with both current and NextGen prototype operations. Their feedback was supplemented with those from the study participants who provided feedback on the acceptability of the BCs after working each traffic scenario with BCs. In the following section, we summarize what we have learned and describe a number of factors to consider in designing acceptable sectors and implementing them through BCs. Due to the qualitative nature of the feedback, we cannot conclusively state which of these factors have greater impact than others. Nevertheless, they may provide valuable information for anyone interested in developing feasible sector designs in FAM and therefore are documented in this paper.

\section{A. Boundary Change Considerations}

When a controller manages traffic within a particular sector, s/he needs to be familiar with the sector and its traffic characteristics. Examples of these characteristics include:

- Geographic information - nearby airports, sector location (e.g., latitude/longitude), elevation, etc.

- Other sector information - waypoints and routes in the sector, names and identifications (IDs) of the neighboring sectors, radio frequencies of one's own and neighboring sectors, etc.

- Traffic information - normal traffic density and flow pattern, such as the number and characteristics of climbing/descending aircraft, overall traffic complexity, major ingress (where traffic enters a sector) and egress (where traffic exits a sector) points, merge points for the traffic flowing through the sector, destination airports, upstream sector(s) that will hand off the aircraft to the sector, downstream sector(s) where the aircraft will be handed off next, etc.

During difficult BCs, controllers can lose situation awareness of the sector and the traffic that is in the new sector. The following section describes some of the factors that seemed to impact controllers' ability to maintain situation awareness of the traffic.

\section{Changes in the spatial relationship between neighboring sectors}

When managing traffic in a particular sector, controllers need to take account of where that sector is located in relation to other surrounding sectors. Figure 5 below illustrates a BC that may create difficulties for controllers during the change.

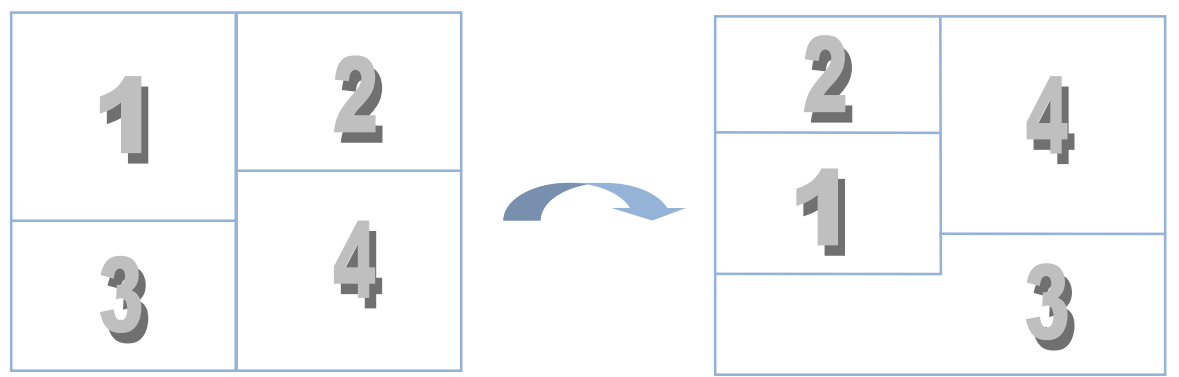

Figure 5. Changes in the spatial relationship between sectors after a boundary change

6

American Institute of Aeronautics and Astronautics 
In this example, Sector 1 has Sector 2 on its right, Sector 3 below, and a small boundary region in common with Sector 4 prior to a BC. The spatial relationship between the sectors changes significantly after the BC such that Sector 2 is now above Sector 1, with Sector 4 to its right.

This type of $\mathrm{BC}$ can be cognitively challenging for controllers for at least two reasons. First, it is likely that controllers form a static spatial mental model of the relationship between sectors and rely on visuo-spatial cues to situate themselves on their displays, both of which - model and cue - need to be updated after a drastic BC. Second, controllers likely form dynamic spatial mental models of the traffic flows through their sector and anchor these models to their overall static cognitive map of their airspace. These dynamic models must also be revised when a BC alters the upstream/downstream relationship between sectors for a given traffic flow. This idea is elaborated further in the next section.

\section{Changes in the upstream/downstream relationship between sectors}

When managing traffic in a particular sector, controllers need to become familiar with normal traffic patterns in that sector. The sector generally has predictable traffic ingress and egress points where aircraft enter and exit the sector. The traffic can also have a set of merge points where multiple traffic streams come together, requiring those points to be monitored closely for potential separation issues.

An important part of familiarizing oneself with sector ingress/egress points for the traffic is learning which upstream sector(s) handoff aircraft at the ingress points and which downstream sector(s) receive a handoff of aircraft at the egress points. When a BC changes the spatial relationship between neighboring sectors, it is also likely to change the upstream/downstream relationship. In the example in Figure 6 below, the dominant traffic flow prior to the BC is from Sector 2 to Sector 1. After the BC, Sector 4 instead of Sector 2 is upstream of Sector 1, while Sector 2 still manages the same flow but over different airspace (that used to belong to Sector 1) and now inherits that flow from new upstream Sector 4. Such drastic changes in the handoff relationship can increase the amount of time necessary for controllers to revise their cognitive spatial maps and attain new situation awareness.

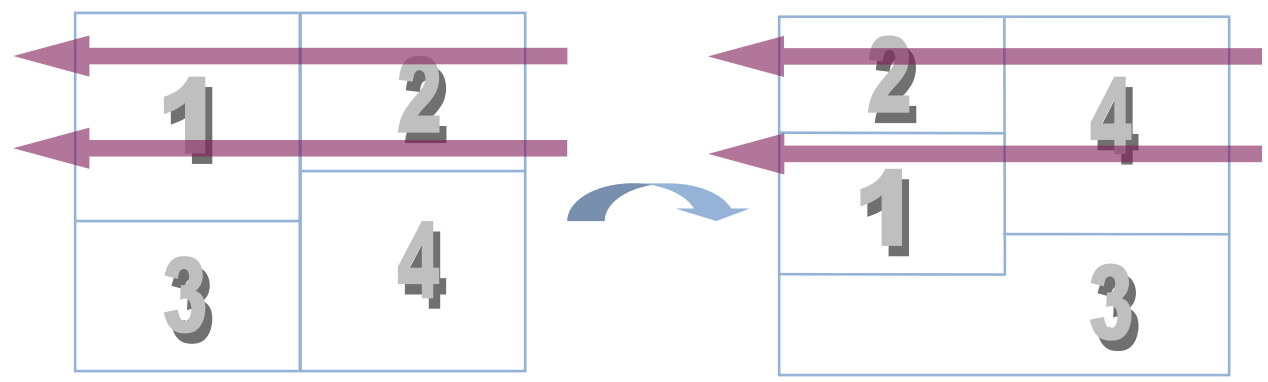

Figure 6. Changes in upstream/downstream relationship between sectors

\section{Inheriting new traffic streams during boundary changes}

When managing traffic in a particular sector, controllers generally monitor an "area of interest" that is slightly upstream of the physical sector. In this region, the controller monitors aircraft that are incoming to the sector in order to accept the handoff and gain situation awareness of the aircraft. Certain BCs result in a sector inheriting a new stream of traffic that was not part of the sector's traffic flow prior to the BC, as illustrated in Figure 7.

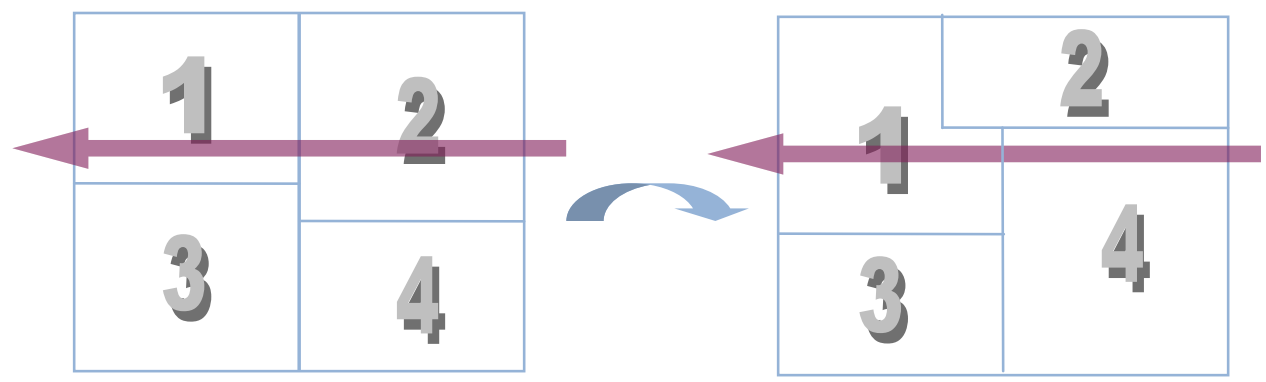

Figure 7. Inheritance of new traffic stream by Sector 4

7

American Institute of Aeronautics and Astronautics 
Here, a traffic stream that originally passed through Sectors 2 and 1 will instead be handed off to Sector 4 due to the BC. Since the aircraft in the traffic stream originally did not pass through Sector 4, the controller in Sector 4 will need more time to become familiar with the new incoming traffic.

\section{Shift in sector location in relation to merge points}

Another design recommendation for the BCs is to keep the relationship between the sector boundaries and the traffic merge points relatively constant. In particular, splitting the traffic streams across multiple sectors prior to a merge point significantly increases the workload associated with the required coordination for all impacted sectors. In the example in Figure 8, Sector 2 manages both traffic streams that merge in Sector 1 prior to the BC. After the $\mathrm{BC}$, Sector 4 needs to manage one of the streams, requiring significant workload to gain situation awareness of the traffic situation, including an awareness of the downstream merge point with the traffic stream from Sector 2. Also, after the BC, Sectors 2 and 4 would need to continue coordinating with each other to provide proper spacing at the merge point. Additionally, sector designs that created sector boundaries near merge points were considered problematic.

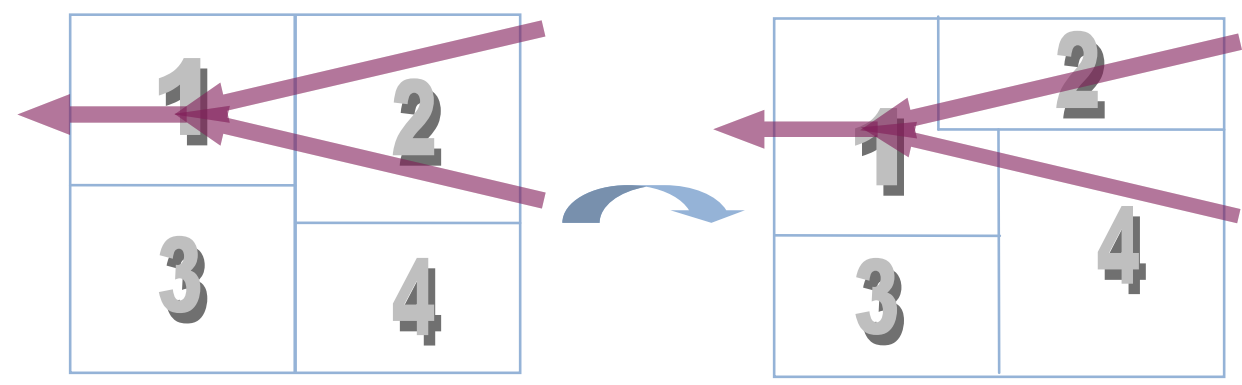

Figure 8. Traffic stream prior to the merge point split between two sectors after the $\mathrm{BC}$

\section{Changes in the range or sector location - need to adjust the scope}

Observations during the simulation as well as subjective feedback afterwards made it clear that certain BCs with large changes in the sector volume or location required adjustments to the display settings, such as the scope range or the X-Y location of the sector map. Figure 9 illustrates an example in which the Sector 1 controller would need to move his/her radar scope to the left and to zoom out on the range in order to cover a larger airspace volume and the new location after the BC. Such changes can potentially add workload by causing controllers to attend to changing their display settings in the middle of the $\mathrm{BC}$ and thus distract them from the traffic management task. The changes may also disrupt their situation awareness because they would have to completely reorient themselves each time the scope needed adjustment.
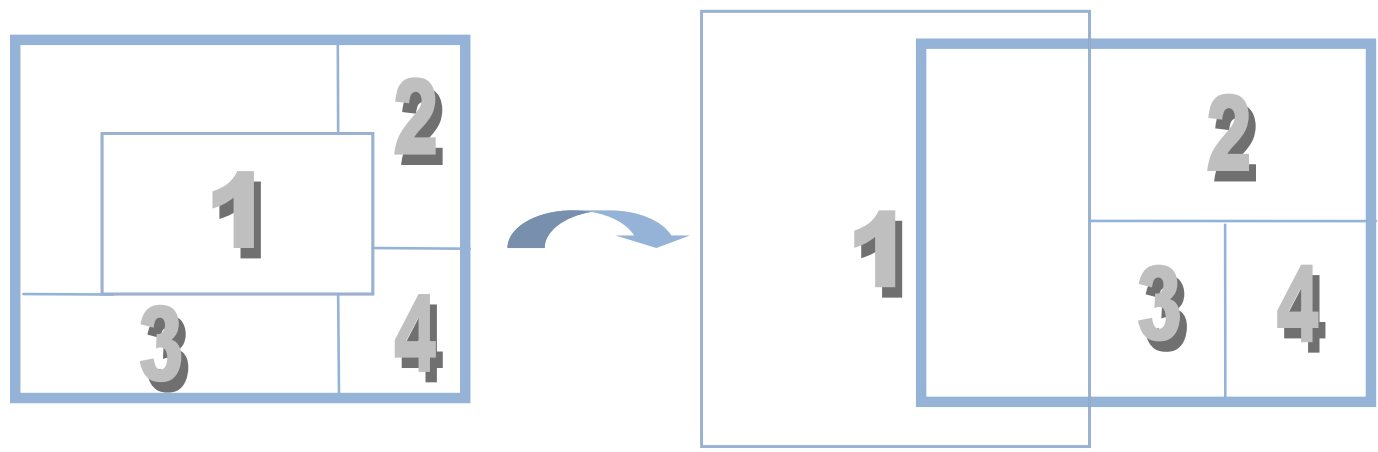

Figure 9. Large change in size and location of Sector 1

\section{B. Sector Design Considerations}

Qualitative feedback from the SMEs and the participants identified several other sector design characteristics that impacted their ability to manage the traffic in the sectors, independent of the $\mathrm{BC}$ operation. We describe below the top four factors that were mentioned.

8

American Institute of Aeronautics and Astronautics 


\section{Thin sectors}

"Thin" sectors, such as the one illustrated in Figure 10, posed significant problems for the controllers. Aircraft that flew across the short axis had very short transit times, creating significant workload by requiring frequent handoffs and pointouts. In certain extreme cases, the controllers needed to hand off the aircraft to the downstream sector before the aircraft even entered their own sector, due to the lack of transit time.

Aircraft that flew along the long axis of a thin sector posed a different problem. The aircraft had very little maneuvering room within the sector if it needed to be taken off the original path. Maneuvering the aircraft outside of the sector (as shown by dashed blue arrow in Figure 10) significantly increased controller workload since the maneuver required coordination with the adjacent sector.

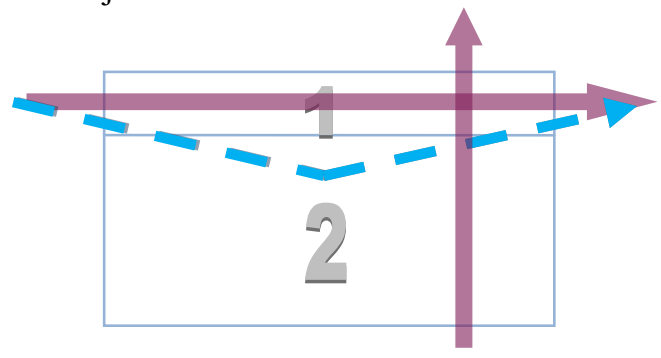

Figure 10. Traffic flow along and across a "thin" sector with little maneuverability and short transit time

\section{Sectors with unusable airspace}

Certain airspace reconfiguration algorithms that were used created unusable airspace. As illustrated in Figure 11, some algorithms created "jagged" sector boundaries (e.g. Sector 1) while others created one or more unusable corners/areas of the airspace (as shown in the boundary between Sectors 2 and 3). In both cases, aircraft that fly through this airspace (shown in red arrows in Figure 11) would require excessive pointouts and coordination. In the simulation, controllers in general kept aircraft away from these boundaries, effectively making them "no fly" zones.

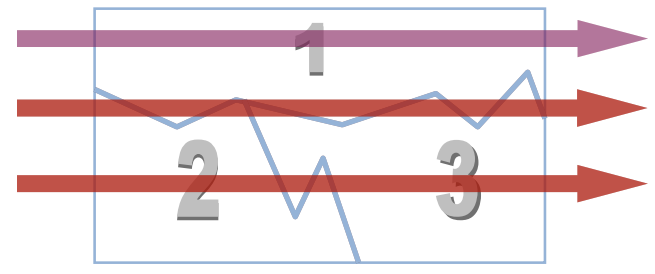

Figure 11. An example of sectors with unusable airspace

\section{Four or more sectors adjoining near a single point}

After each simulation run, we presented the sector designs that were used during the run to the controller participants as part of their post-run questionnaire packet. We asked them to re-draw the boundaries to improve them, if necessary. One common correction was to the modify sector boundaries where four sectors had joined at or near a single point/corner (a "4-way corner") into a new design that moved the corners such that only three sectors shared the same corner.

The main reason for the correction appears to be that controllers wanted to avoid situations in which traffic traverses through or near four sectors at the same time, which may require a handoff and two pointouts for a single aircraft. The left figure in the Figure 12 illustrates such a situation. In contrast, in a 3-way corner, traffic that traverses three sectors near one corner requires only one handoff and one pointout, and traffic that traverses two sectors requires only a handoff. This amount of coordination is manageable and is done regularly in current operations (see the right figure in the Figure 12) 

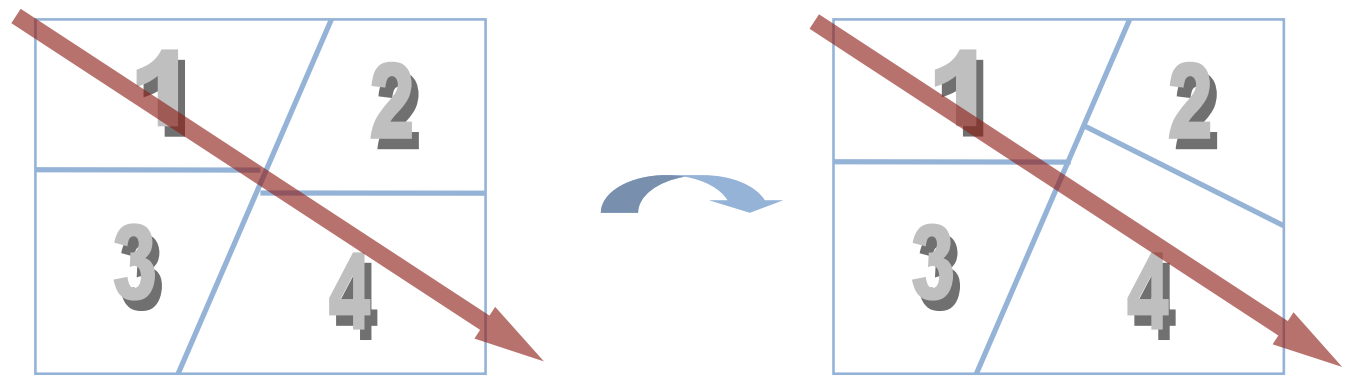

Figure 12. An example of traffic flow across four or three sectors near one corner of airspace

\section{Conclusion}

The 2009 study focused on the impact of airspace reconfiguration on controllers. The overall results were encouraging. Even though the controller participants were faced with a challenging BC environment - e.g., high BC frequency, high traffic volume, fairly large change of aircraft ownership, fairly large volume change, short transition time (i.e., 3-minute preview prior to the $\mathrm{BC}$ ), etc. - they were able to handle most of the BCs effectively. They commented that they could handle large volume changes and severe BCs if they were given sufficient transition time from one configuration to the next. However, they also emphasized that they could not maintain situation awareness of the aircraft during the BCs and had to rely heavily on the automated conflict detection to help them monitor the aircraft safety during the BCs.

Along with data that correlate number of aircraft, aircraft that change ownership, and volume gained/lost with controller workload and acceptability, subjective feedback provided a rich description of sector design and boundary change considerations to be adopted in Flexible Airspace. Some of these considerations result from human cognitive limitations in quickly gaining situation awareness and anchoring their traffic situational models to airspace features in a time-critical situation. Other considerations arise from the nature of air traffic control which results in excessive workload and coordination when the sector design fails to provide sector boundaries that function harmoniously with the traffic flow.

While the 2009 study examined the human-system integration issues related to controllers workload and acceptability, we did not examine the role of the ANSP in design, assessment, and implementation of airspace reconfiguration. In August 2010, we have conducted a follow-up study to examine the roles, procedures, and tools needed to assess and implement FAM. We have examined the team configuration consisting of members from TMU and control floors (e.g., supervisors and controllers) and explored where the FAM function resides. We have also examined the benefits of FAM in weather scenarios. Airspace was reconfigured only once every hour and was done to relieve larger and more sustained traffic congestions than in 2009 study. The data from this study have been collected and is currently being analyzed.

In sum, there is an exciting potential of utilizing FAM in TBO airspace with advanced decision support tools and Data Comm. Continued efforts to explore the benefits and feasibility in the NextGen operational context can provide a significant value to the future of the air transport system.

\section{Reference}

${ }^{1}$ Joint Planning and Development Office. Concept of Operations for the Next Generation Air Transportation System, version 2.0., 2007.

${ }^{2}$ Kopardekar, P., Bilimoria, K. and Sridhar, B. Airspace Configuration Concepts for Next Generation Air Transporation, Air Traffic Control Quarterly, Vol 16 [4]., 2008.

${ }^{3}$ Zelinski, S. A Comparison of Algorithm Generated Sectorizations. Eighth USA/Europe Air Traffic Management Research and Development Seminar (ATM 2009), Napa, CA., 2009.

${ }^{4}$ Brinton, C. and Pledgie, S. Airspace Partitioning using Flight Clustering and Computational Geometry. In Proceedings of the 27th Digital Avionics Systems Conference (DASC), St. Paul, MN., 2008.

${ }^{5}$ Klein, A., Rogers, M. and Kaing, H. Dynamic FPAs: A New Method for Dynamic Airspace Configuration. Integrated Communications Navigation and Surveillance (ICNS) Conference. Bethesda, MD., 2008.

${ }^{6}$ Yousefi, A., Khorrami, B., Hoffman, R. and Hackney, B. Enhanced Dynamic Airspace Configuration Algorithms and Concepts, Metron Aviation Inc., Technical Report No. 34N1207-001-R0., 2007.

${ }^{7}$ FAA ATO Planning, Research and Technology. An Operational Concept for Mid-Term High Altitude (High PerformanceBased) Airspace, draft version 4.17., 2010.

10

American Institute of Aeronautics and Astronautics 
${ }^{8}$ Homola, J., Lee, P. U., Prevot, T., Lee, H., Kessell, A., Brasil, C. and Smith, N. A Human-in-the-Loop Exploration of the Dynamic Airspace Configuration Concept, AIAA Guidance, Navigation, and Control (GNC) Conference and Exhibit, Toronto, Canada., 2010.

${ }^{9}$ Lee, P. U., Prevot, T., Homola, J., Lee, H., Kessell, A., Brasil, C. and Smith, N. Impact of Airspace Reconfiguration on Controller Workload and Task Performance, $3^{\text {rd }}$ Intl. Conference on Applied Human Factors and Ergonomics, Miami, FL., 2010.

${ }^{10}$ Jung, J., Lee, P. U., Kessell, A., Homola, J., and Zelinski, S. Effect of Dynamic Sector Boundary Changes on Air Traffic Controllers, AIAA Guidance, Navigation, and Control (GNC) Conference and Exhibit, Toronto, Canada., 2010. 